\title{
LYSO modules for the JEDI polarimeter: production, laboratory tests and results of first measurements
}

\section{Shergelashvili*1, D. Mchedlishvili ${ }^{1,2}$, F. Müller ${ }^{3,4}$, I. Keshelashvili ${ }^{4}$ (for the JEDI collaboration)}

${ }^{1}$ SMART | EDM_Lab, Tbilisi State University, 0179 Tbilisi, Georgia

${ }^{2}$ High-Energy Physics Institute, Tbilisi State University, 0186 Tbilisi, Georgia

${ }^{3}$ Physics Institute III B, RWTH Aachen, Otto-Blumenthal-Strasse, 52074 Aachen, Germany

${ }^{4}$ Institute of Nuclear Physics, Forschungszentrum Jülich, 52425 Jülich, Germany

E-mail: shergelashvili.dito@gmail.com

\begin{abstract}
The JEDI ${ }^{\dagger}$ collaboration aims to search for the electric dipole moment (EDM) for the protons and deuterons in the storage ring ( $\mathrm{sEDM}$ ). One of the main components of the srEDM experimental set-up is the polarimeter to observe the slow buildup of vertical polarization. In our concept, the polarimeter is based on inorganic novel LYSO crystals and low voltage silicon photomultipliers (SiPM). Up to now, more than 30 modules have been assembled in different configurations. They have first been tested in the laboratory with internal and external radiation sources and subsequently, they have been exposed to the experimental environment with accelerator beams. We have performed three test beam times at several different beam energies. The results of the measurements and accumulated experience of the module production will be presented.
\end{abstract}

XVII International Workshop on Polarized Sources, Targets \& Polarimetry

16-20 October 2017

Kaist, South Korea

\footnotetext{
* Speaker.

${ }^{\dagger}$ Electric Dipole moment Investigation
} 


\section{Introduction}

The JEDI collaboration was formed to exploit COSY (COoler SYnchrotron) facility and make all necessary investigation toward the design of dedicated storage ring which aims to search Electric Dipole Moment (srEMD) of charged particles [1]. The key challenge for srEDM is the provision of a sensitive and efficient method to determine the tiny change of the beam polarization direction. The new concept of the dedicated polarimetry (target, detection system, data acquisition), will allow to push the EDM sensitivity level by orders of magnitude in a future storage ring [3, 4].

According to the requirement, the polarimetry development consists of two main parts: target and detector system constructions [5]. Current experiments are focused on the defelopment of the calorimeter part of the detector. The material for the new calorimeter is LYSO (Lutetiumyttrium oxy orthosilicate) scintillator crystal because of its special properties, such as density, high light output, short decay time [2]. Scintillation light readout is made by a SiPM array (Silicon PhotoMultiplier), which generates an analog signal. This signal needs to be digitized for data recording and further analysis. The Following flow chart shows the basic working principle of the polarimeter: Particle deposits energy in the crystal and proportional numbers of photons are emitted. The SiPM registers photons and generates the analog signal. The output of Multiple modules are digitized by the fast ADCs and the digital information is distributed via $10 \mathrm{Gbit} / \mathrm{s}$ network to the server computer. The server computer records and sends data stream for online analysis. High stability ( $<2 \mathrm{mV}$ deviation for long time measurement) and low noise power supply is designed specifically for the SiPM. Supply voltage range is 25-31 volt and can be set according to the experiment setup.

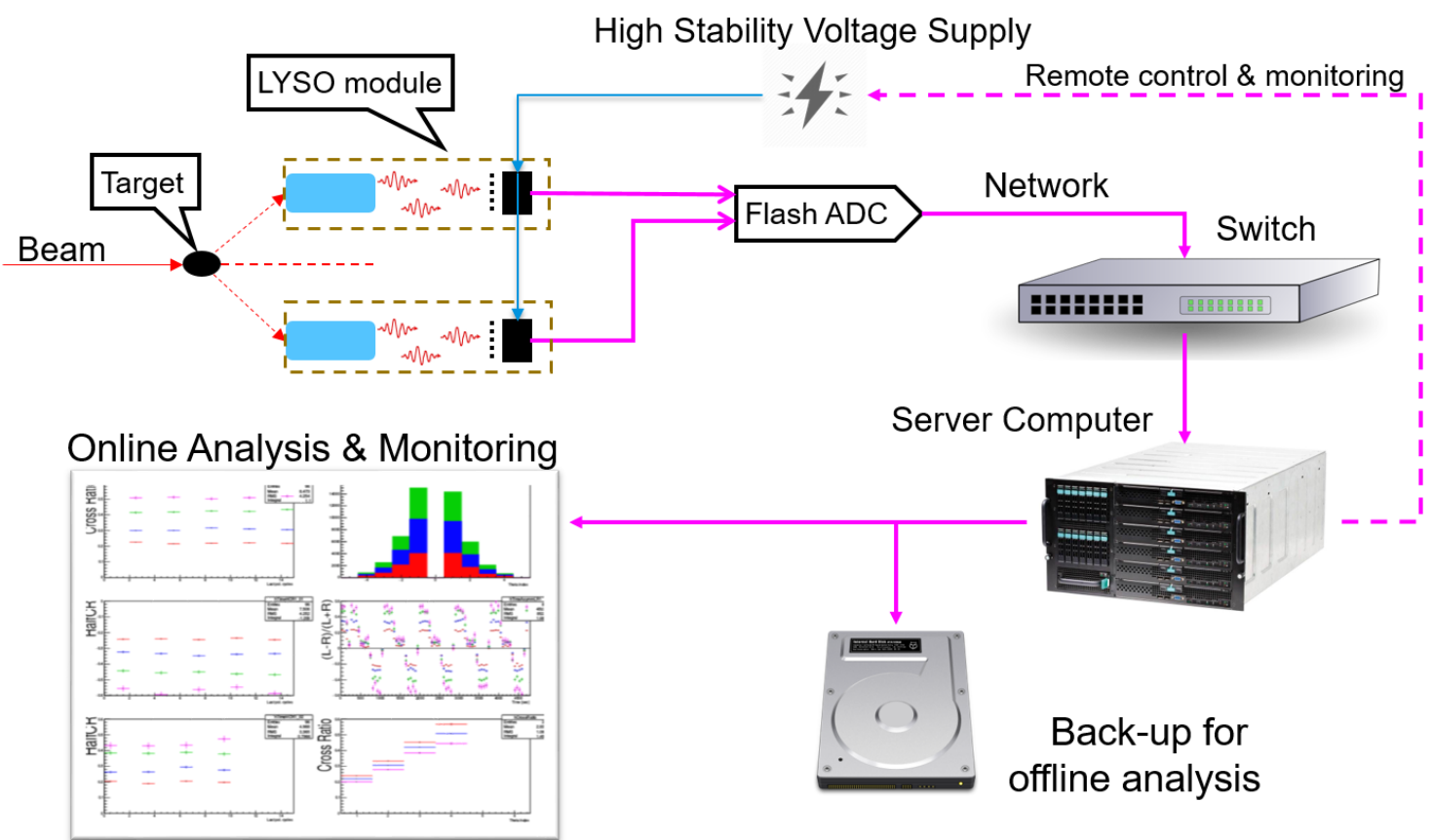

Figure 1: General view of the JEDI polarimetry DAQ. 


\section{LYSO Module Assembling}

The LYSO module design for the JEDI polarimetry was created in Juelich research center. At the starting point, different constructions and materials were tested. After considering the multiple laboratory tests we established the final version. The main parts of each moduleare LYSO crystal and SiPM sensor board. Both of them are manufactured according to dedicated polarimetry necessity. The crystal is $8 \mathrm{~cm}$ long with transverse sizes of $3 \times 3 \mathrm{~cm}^{2}$. This length is sufficient to stop deuterons of up to $300 \mathrm{MeV}$ kinetic energy. The specific Brag peak is located at about $6 \mathrm{~cm}$ deep for the $270 \mathrm{MeV}$ deuterons (experimental result). The geometry of the crystal allows for the horizontal flipping. The idea behind this is to double the lifetime for the most expansive part of the calorimeter with the very simple method. Besides, all 8 vertices are cut symmetrically and can hold aluminum housing from both sides.

The second crucial part is SiPM $3 \times 3 \mathrm{~cm}^{2}$ board. Each board consist of 64 pieces of SensL J-Series chips with $14 \mathrm{~K}-20 \mu \mathrm{m}$ pixels. In total $900 \mathrm{k}$ pixels are responsible for getting photons from one LYSO crystal [6]. The SiPM board has surface-mounted connector and can be easily attached to the module PCB. This PCB has several passive components and manages power input and signal out rails. LYSO module components and the final assembly is shown on the following picture:

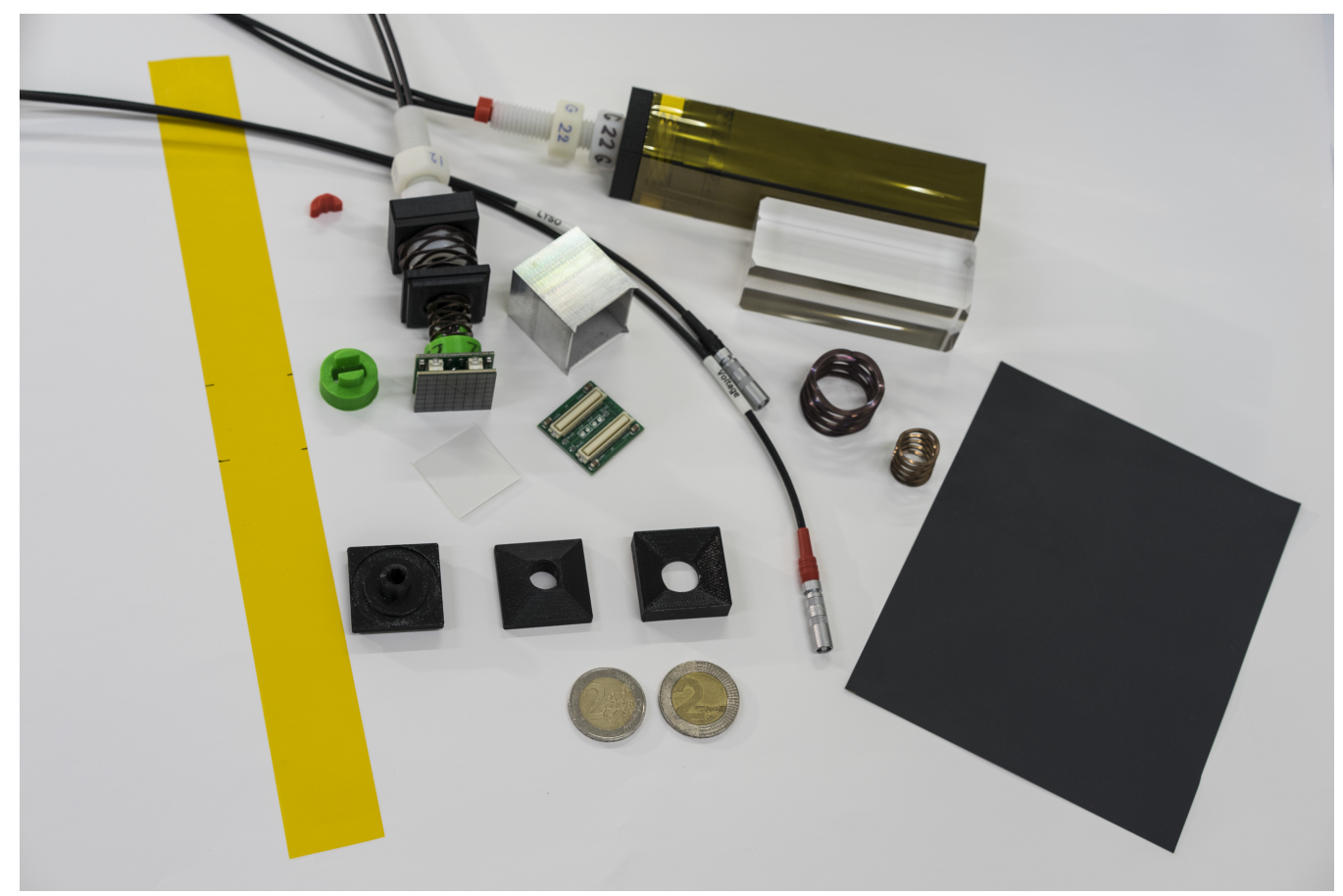

Figure 2: LYSO module and it's content.

Tight connection between the LYSO crystal and the SiPM board is made by using special parts from a different materials which create whole module body. The module integrity is guaranteed by $3 \mathrm{D}$ printed plastic materials, aluminum housing, springs and foils. In between the SiPM board 
and the crystal, $1 \mathrm{~mm}$ thick silicon pad is installed, which improves the optical contact with the SiPM and also softens the mechanical tension between the two surfaces. Besides, it protects the SiPM pixels from the direct internal radiation of the LYSO crystal. Room light tightness is very important to avoid wrong measurements which is realized with $50 \mu \mathrm{m}$ tedlar foil. The tedlar is wrapped around the teflon foil which is responsible for light reflection and leads to higher light output. The mechanical fixation and construction stability are ensured by a kapton foil. The amount of building materials around the module is minimized. This makes the module easy to install with different configurations and introduces minimal energy loss for the incident particles (less that 1\%) through the foils.

\section{LYSO Module Lab inspection}

Quality and performance check is a foundational principle for any production. After successful assembling, the special tests are performed for the modules before installing them in the experimental area. The laboratory for LYSO module inspection is equipped with special test bench including portable DAQ, power supply, radioactive sources, etc. The portable DAQ is based on Raspberry Pi computer and Red Pitaya. The DAQ software is based on CERN ROOT and gives opportunity to make fast online data analysis. The test procedure includes several steps. The intermediate test includes powering up and voltage tuning for each individual module to achieve best performance (high gain, good signal to noise ratio). The electrical characteristics of the SiPM's array and the connector board (including a wiring and passive components) were checked by the micro ammeter. The ammeter measures the leakage current in the bypass capacitors (must be $<1$ $\mu \mathrm{A}$ ) and the "dark current" through the SiPM chips (must be $<10 \mu \mathrm{A}$ ) while the supply voltage is $30 \mathrm{~V}$. In the next step a real measurement takes place using DAQ. Before using the radioactive sources, light tightness measurement is performed in order to ensure wrapping quality.

The next experiments aim to check LYSO module performance together with light-sensitive parts. For that, two different ${ }^{60} \mathrm{Co}$ and ${ }^{22} \mathrm{Na}$ sources were used separately. The results after data processing and fitting, are presented in the following figures:
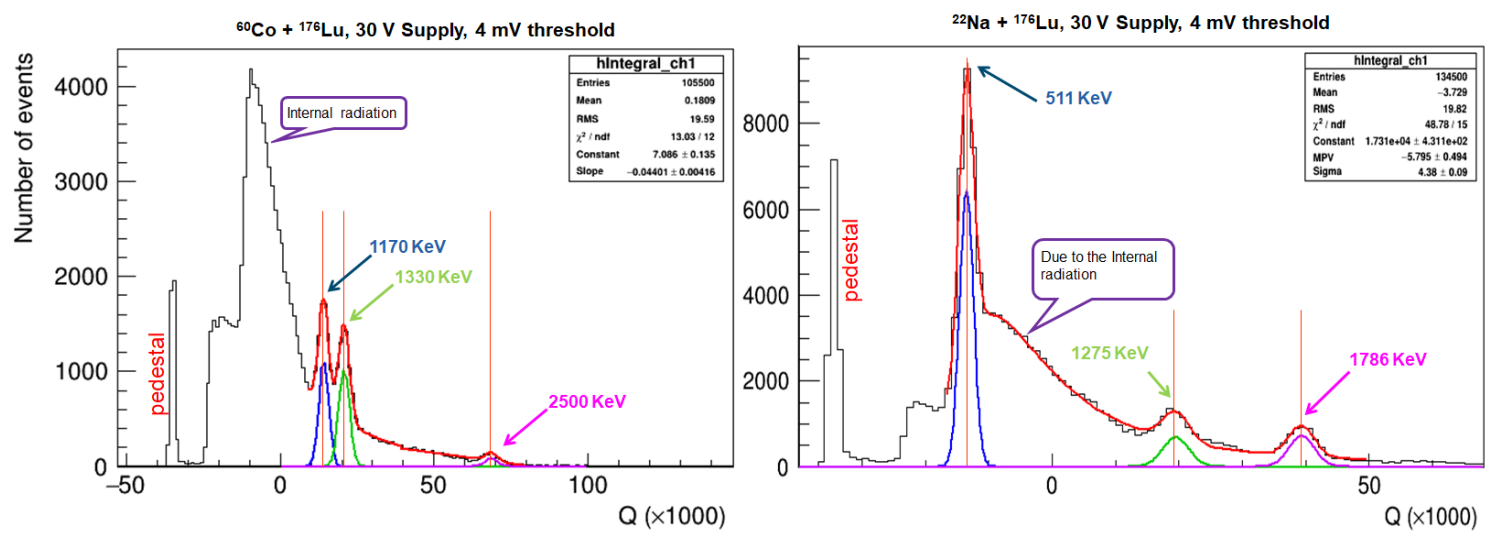

Figure 3: LYSO module performance results on different radiactive sources.

The left figure presents $\beta^{-}$decay for ${ }^{60} \mathrm{Co}$ with two consecutive transactions to the main state. The first transaction corresponds to the $1330 \mathrm{KeV}$ energy, and the second one to $1170 \mathrm{KeV}$, while 
the simultaneous registration of the two $\gamma$ 's is marked with $2500 \mathrm{KeV}$. On the right side, $\beta^{+}$decay takes place with three $\gamma$ emission (one $1275 \mathrm{KeV}$ and two $511 \mathrm{KeV}$ ) for the ${ }^{22} \mathrm{Na}$. The spectra also show coincidence in the LYSO $(1786 \mathrm{KeV})$. On both plots, ${ }^{176} \mathrm{Lu}$ radiation is detectable which we called LYSO internal radiation. Besides that, modules are not calibrated, the energy peaks nicely define decay properties radioactive isotopes. After extracting the peak positions and the widths, we can check module linearity at the low energy region. The next figure shows LYSO module linearity result together with the corresponding energy resolutions:

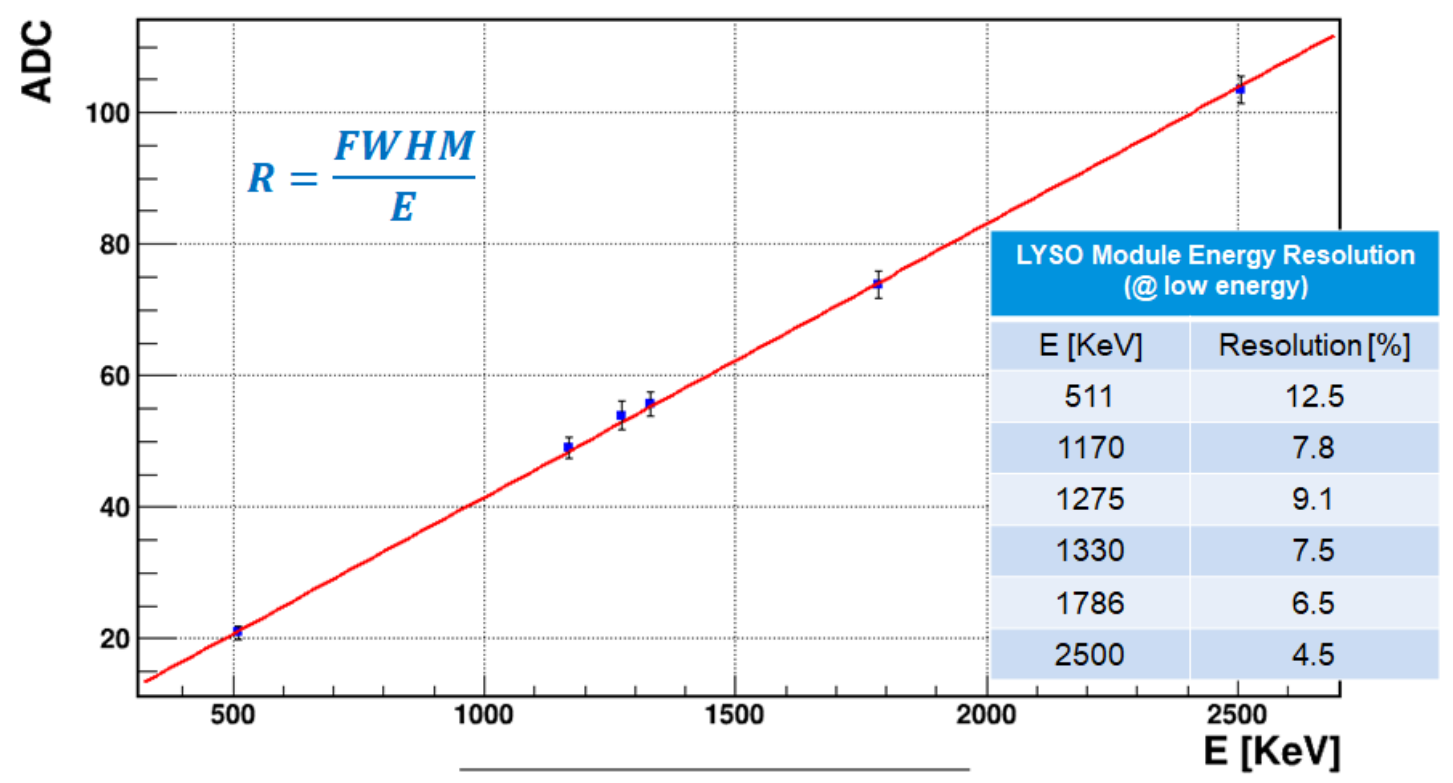

Figure 4: LYSO module linearity test results and resolutions at low energy.

According to the figure, the LYSO module is linear at the low energy region and the resolution is decreasing at higher energes. Data analysis taken during the beam time shows that energy resolution at $270 \mathrm{MeV}$ is below the $1 \%$ [7].

\section{Time Resolution Analysis}

The LYSO module together with the SiPM board generates signal in a volts range and does not require a preamplifier before digitizing. For the data readout, the SIS3316 VME DIGITIZER modules are used from the "Stuck Innovative Systeme", also called Flash ADC. Each module can handle 16 individual channels and can take 250 MSamples/s per channel with 14 bit resolution. The Flash ADC is powered by 5 FPGAs and has DDR3 memory with 2GB capacity [8]. The module has special I/O connectors in order to synchronize data recording for all channels though multiple modules. Besides the hardware, the device also has software features . User can estimate signal trigger time with tens of picoseconds resolution, using the special data registers. This operation also involves integrated Constant Fraction Discriminator function. The time resolution offered by the flash ADC is satisfactory for Time Of Flight (TOF) measurement. The results from successful TOF 
calculation can be used for the energy calibration in a wide range, which leads to higher precision in energy measurement. The first attempt was to do offline analysis on the data which was obtained with deuteron beam. According to the flash ADC configuration all channels were triggered at the same time while source of this trigger could be any individual channel. In this mode the absolute time values of individual channels are not presented in ADC data. Therefore, assumptions have to be made to estimate the number of samples between start and stop and precise time corrections applied using ADC data. The result of this analysis is presented in the following figure:

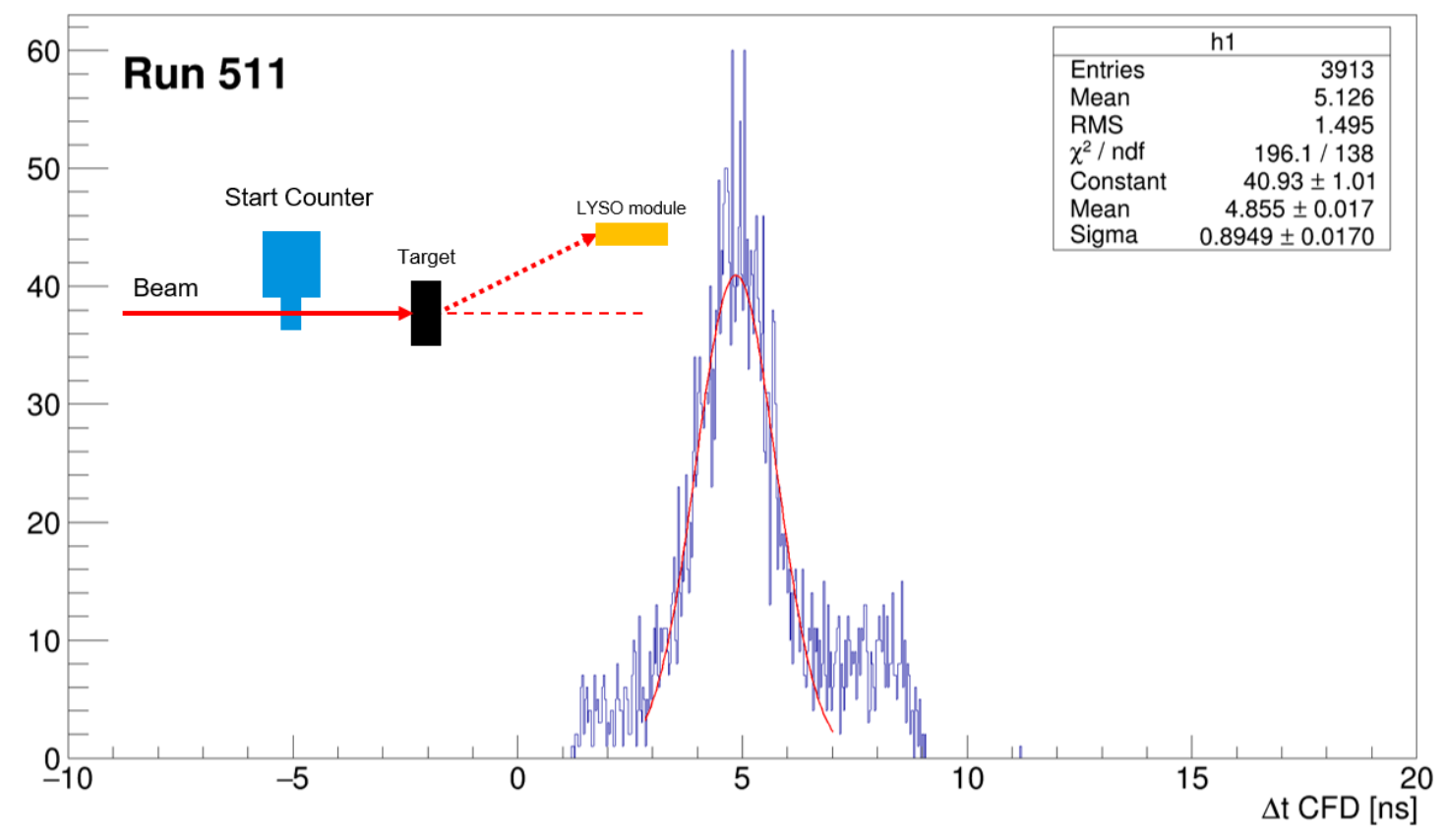

Figure 5: Deuteron TOF between the start counter and the detector.

The TOF of the deuteron is measured in between start counter and one of the LYSO modules. The entries were filtered in the way that only the start counter and the preselected module were filled with data. Deuteron flight distance was 1 meter with the kinetic energy of $270 \mathrm{MeV}$. According to Figure 5, the resolution of TOF is about $1 \mathrm{~ns}$. This is 4 times better resolution than $4 \mathrm{~ns}$ sampling interval, but it is not sufficient for the energy calibration. With the SIS3316 module, a tens of picoseconds time resolution can be achieved in self-triggering configuration, which means that further developments and tweaking of the readout system in order to reach the desired time resolution.

\section{Acknowledgements}

This work has been financially supported by the grants from the Shota Rustaveli National Science Foundation of the republic of Georgia (SRNSF Grant agreement 04/01, "Search for Electric Dipole Moments using Storage Rings (srEDM)"; SRNSF Grant No. 217854, "A firstever measurement of the EDM of the deuteron at COSY"). 


\section{References}

[1] JEDI Collaboration, http://collaborations.fz-juelich.de/ikp/jedi

[2] C. Melcher and J. Schweitzer, IEEE Trans. Nucl. Sci. 39, 502 (1992)

[3] D. F. Nelson, A. A. Schupp, R. W. Pidd, and H. R. Crane, "Search for an Electric Dipole Moment of the Electron", Phys. Rev. Lett., vol. 2, pp. 492-495, (1959)

[4] J. Pretz, "Measurement of Permanent Electric Dipole Moments of Charged Hadrons in Storage Rings", Hyperfine Interact., vol. 214, no. 1-3, pp. 111-117, (2013)

[5] I. Keshelashvili "Towards JEDI Polarimetry", XVIth International Workshop in Polarized Sources, Targets, and Polarimetry, PoS(PSTP2015)026

[6] https://www.sensl.com/downloads/ds/TN\%20-\%20Intro\%20to\%20SPM\%20Tech.pdf

[7] F. Müller "EDM Polarimeter Development at COSY for the JEDI Collaboration", 2018

[8] http://www.struck.de/sis3316.html 